\title{
Reply to: A key role for vitamin D binding protein in COVID-19?
}

\author{
Mehran Arab-Ahmadi ${ }^{1}$ Behdad Behnam ${ }^{2}$ - Alireza Abrishami ${ }^{3}$ - Morteza Sanei-Taheri ${ }^{4,5}$
}

Accepted: 17 December 2020 / Published online: 12 February 2021

(c) Springer-Verlag GmbH, DE part of Springer Nature 2021

Keywords COVID-19 - Vitamin D · Vitamin D binding protein · Outcome

\section{Dear Editor,}

We appreciate the interest in our article investigating the association of vitamin D level with severity and outcome of COVID-19 [1]. Our primary goal was to evaluate the prognostic role of serum vitamin $\mathrm{D}$ concentration on the extent of lung involvement and final outcome in patients with COVID-19.

In patients with COVID-19 pneumonia, a hyperinflammatory syndrome with activation of the complements system may be involved in progression of the disease to acute respiratory distress syndrome (ARDS). The C5a-C5aR axis plays an important role in progression of the disease to ARDS. Vitamin D binding protein (DBP) release augments the chemotactic effect of complement derived C5a and C5a des Arg, leading to a cascade of inflammatory responses [2]. 25(OH)D3 and 1,25(OH)2D3 compete for the same binding site on DBP and so may inhibit this chemotaxis [3]. A

This Reply refers to the Letter to the Editor available at: https:// doi.org/10.1007/s00394-020-02479-8.

The original publication is available at: https://doi.org/10.1007/ s00394-020-02411-0.

\section{Alireza Abrishami}

abr.alireza@yahoo.com

1 Department of Radiology, Advanced Diagnostic and Interventional Radiology Research Center, Tehran University of Medical Sciences, Tehran, Iran

2 Departments of Internal Medicine, Firoozgar Hospital, Iran University of Medical Sciences, Tehran, Iran

3 Department of Radiology, Shahid Labbafinejad Hospital, Shahid Beheshti University of Medical Sciences, Tehran, Iran

4 Department of Radiology, Shohada Tajrish Hospital, Shahid Beheshti University of Medical Sciences, Tehran, Iran

5 President of Iranian Society of Radiology, Tehran, Iran recent study by Batur et al. [4] suggested that variations in the prevalence of COVID-19 and its mortality rates among countries may be explained by vitamin D metabolism differed by the DBP polymorphism of rs7041 and rs4588. Although our study did not investigate the role of DBP in COVID-19 severity, our results and those of other studies in other countries, such as Weir et al. in USA (5), confirmed the role of vitamin D level in COVID-19 severity and outcomes. These studies provided new evidence for clinicians and health policy makers to consider vitamin D supplementation for the improvement of clinical outcome of patients with COVID-19. However, currently we need to expand our knowledge on the mechanism of the association of vitamin D and COVID-19 especially by studying the role of DBP. Hence, further studies in this field are highly recommended.

\section{References}

1. Abrishami A, Dalili N, Torbati PM, Asgari R, Arab-Ahmadi M, Behnam B et al (2020) Possible association of vitamin D status with lung involvement and outcome in patients with COVID-19: a retrospective study. Eur J Nutr. https://doi.org/10.1007/s0039 4-020-02411-0

2. Raymond M-A, Désormeaux A, Labelle A, Soulez M, Soulez G, Langelier Y et al (2005) Endothelial stress induces the release of vitamin D-binding protein, a novel growth factor. BiochemBiophys Res Commun 338(3):1374-1382

3. Speeckaert MM, Speeckaert R, Delanghe JR (2020) Vitamin D binding protein in COVID-19. Clin Med 20(5):E136-E137

4. KarciogluBatur L, Hekim N (2020) The role of DBP gene polymorphisms in the prevalence of new coronavirus disease 2019 infection and mortality rate. J Med Virol. https://doi.org/10.1002/ jmv.26409

5. Weir EK, Thenappan T, Bhargava M, Chen Y (2020) Does vitamin D deficiency increase the severity of COVID-19? Clin Med 20:e107-e108 